\title{
Beyond the operating room: do hospital characteristics have an impact on surgical site infections after colorectal surgery? A systematic review
}

Rui Malheiro ${ }^{1,3^{*}} \mathbb{B}$, Bárbara Peleteiro ${ }^{1,2,3}$ and Sofia Correia ${ }^{1,2,3}$

\begin{abstract}
Background: Hospital characteristics have been recognized as potential risk factors for surgical site infection for over 20 years. However, most research has focused on patient and procedural risk factors. Understanding how structural and process variables influence infection is vital to identify targets for effective interventions and to optimize healthcare services. The aim of this study was to systematically review the association between hospital characteristics and surgical site infection in colorectal surgery.
\end{abstract}

Main body: A systematic literature search was conducted using PubMed, Scopus and Web of Science databases until the 31st of May, 2021. The search strategy followed the Participants, Exposure/Intervention, Comparison, Outcomes and Study design. The primary outcome of interest was surgical site infection rate after colorectal surgery. Studies were grouped into nine risk factor typologies: hospital size, ownership affiliation, being an oncological hospital, safetynet burden, hospital volume, surgeon caseload, discharge destination and time since implementation of surveillance. The STROBE statement was used for evaluating the methodological quality.

A total of 4703 records were identified, of which 172 were reviewed and 16 were included. Studies were published between 2008 and 2021, and referred to data collected between 1996 and 2016. Surgical site infection incidence ranged from 3.2 to $27.6 \%$. Two out of five studies evaluating hospital size adjusted the analysis to patient and procedure-related risk factors, and showed that larger hospitals were either positively associated or had no association with SSI. Public hospitals did not present significantly different infection rates than private or non-profit ones. Medical school affiliation and higher safety-net burden were associated with higher surgical site infection (crude estimates), while oncological hospitals were associated with higher incidence independently of other variables. Hospital caseload showed mixed results, while surgeon caseload and surveillance time since implementation appear to be associated with fewer infections.

Conclusions: Although there are few studies addressing hospital-level factors on surgical site infection, surgeon experience and the implementation of a surveillance system appear to be associated with better outcomes. For hospitals and services to be efficiently optimized, more studies addressing these variables are needed that take into account the confounding effect of patient case mix.

*Correspondence: rui.coelho@arsnorte.min-saude.pt

${ }^{1}$ EPIUnit-Instituto de Saúde Pública, Universidade do Porto, Rua das

Taipas 135, 4050-091 Porto, Portugal

Full list of author information is available at the end of the article

(c) The Author(s) 2021. Open Access This article is licensed under a Creative Commons Attribution 4.0 International License, which permits use, sharing, adaptation, distribution and reproduction in any medium or format, as long as you give appropriate credit to the original author(s) and the source, provide a link to the Creative Commons licence, and indicate if changes were made. The images or other third party material in this article are included in the article's Creative Commons licence, unless indicated otherwise in a credit line to the material. If material is not included in the article's Creative Commons licence and your intended use is not permitted by statutory regulation or exceeds the permitted use, you will need to obtain permission directly from the copyright holder. To view a copy of this licence, visit http://creativecommons.org/licenses/by/4.0/. The Creative Commons Public Domain Dedication waiver (http://creativeco mmons.org/publicdomain/zero/1.0/) applies to the data made available in this article, unless otherwise stated in a credit line to the data. 
Keywords: Characteristics, Colorectal surgery, Facilities, Hospital, Review, Surgical site infection, Surveillance

\section{Background}

Surgical site infection (SSI) is the third most common healthcare-associated infection (HAI) [1], and it is known to have a high impact on hospital length of stay, expenditure, surgical morbidity and mortality [1-3]. According to the latest report from the European Centre for Disease Prevention and Control, open colon surgery was the procedure associated with the highest risk of SSI (10.1 per 100 operations) followed by laparoscopic colon surgery (6.4 per 100 operations) [4]. Given its burden, efforts have been made to identify modifiable risk factors. In their guideline for the prevention of SSI in 1999, the Centers for Disease Control and Prevention (CDC) acknowledged that the risk of SSI is influenced by the characteristics of the patient, procedure, personnel and hospital [5]. Based on the same rationale, the National Healthcare Safety Network (NHSN), the North American surveillance system for HAI, combine facility, patient and procedure-level variables in their SSI risk adjustment models to predict the number of expected infections [6]. However, most research has focused on patient and procedural risk factors. Similarly, preventive interventionseither isolated or in a bundle-have focused exclusively on optimizing patient condition and delivering the surgical procedure as safely as possible [7-10]. Hospital characteristics have been consistently overlooked. Even though most may be deemed as non-modifiable, they are proxy indicators of unmeasured variables, such as cleanliness, structural and organizational characteristics, staffing or training [11], all of which may be potential targets for improvement. Better structural resources and better processes should provide better outcomes. Thus, understanding how structural and process variables may influence SSI is vital to pinpoint effective interventions and to optimize healthcare services.

The aim of this study was to systematically review the published literature regarding the association between hospitals' characteristics, including services provided, and SSI incidence after colorectal surgery.

\section{Methods}

\section{Search strategy}

The search strategy followed the Participants, Exposure/ Intervention, Comparison, Outcomes and Study (PE/ ICOS) design [12]. PubMed, Scopus and Web of Science databases were searched, with no date limit, using the following query: (colorectal OR colon OR rectal) AND (surgical site infection OR wound infection OR skin infection) AND (effect OR risk OR association OR impact OR relation* OR influence OR outcome). All sources were last searched on May 31st, 2021, and backward citation tracking was conducted for all included articles. This systematic review was undertaken using the PRISMA (Preferred Reporting Items for Systematic Reviews and Meta-analyses) guidelines [13] but was not registered in the PROSPERO database.

\section{Inclusion and exclusion criteria}

The inclusion criteria were as follows: (1) articles written in English, Portuguese, Spanish, Italian, French or German, (2) not a review article, editorial, comment, guideline or descriptive study, (3) patients submitted to colorectal surgery, (4) analysis of risk factors representing hospital characteristics, services or organization (no patient or procedure-related risk factors), (5) SSI as an outcome and (6) studies with odds ratio (OR) or relative risk (RR), or raw data allowing the estimation of those measures of association.

\section{Data extraction}

$\mathrm{RM}$ and BP independently reviewed titles and abstracts of all records retrieved from electronic searches, applying the aforementioned criteria. Any disagreements were solved through a consensus discussion, or involving SC. Full texts and supplement material (when available) of all identified studies were then reviewed by the same researchers. Given that all included studies were observational, the STROBE checklist was used for evaluating their methodological quality [14]. This is a checklist of 22 items that should be included in reports of observational studies. Each sub-item was graded as 1, if the study reported them as recommended; 0 , if the sub-item was missing from the study; or 0.5 , if the sub-item was included but only partially met the recommendation. As some sub-items could be non-applicable, the maximum score ranged from 24 to 30 .

Data on first author, publication year, language, study design, country, recruitment period, surgical procedures considered, procedure codes used, databases used, type and criteria of SSI and study size were retrieved. Missing data was registered as such, and no assumptions were made. Nonetheless, authors were contacted to retrieve necessary data when studies fulfilling the inclusion criteria had missing data. When applicable, information on whether the hospital had an infection control team and whether surveillance included post-discharge diagnosis were also retrieved. 
The primary outcome of interest was SSI rate after colorectal surgery, whether superficial incisional, deep incisional or organ/space, as defined by the CDC [10]. Measures of association and their respective 95\% confidence interval (CI) were retrieved, or estimated when raw data was available.

Studies were grouped in nine risk factor typologies: hospital size, for those studies that estimated the association between hospital's number of beds and SSI; hospital ownership, when the analysis focused on whether hospitals were public, private or non-profit; medical school affiliation, for the comparison of teaching versus non-teaching hospitals; Oncological hospitals, for studies researching whether a hospital being a specialized oncological center had an impact on SSI incidence; safety-net burden, defined as the proportion of patients a hospital treats who are either uninsured or insured by Medicaid, an American state program that helps with healthcare costs for people with limited income and resources; hospital volume of procedures, when the risk factor analyzed was the number of colorectal procedures performed at each hospital; surgeon volume of procedures, when the risk factor was the number of colorectal procedures performed per surgeon, rather than per hospital; postdischarge destination, analyzing if patients discharged to their homes had different outcomes when compared to those discharged to skilled facilities; and surveillance time, for studies estimating the impact of surveillance programs over the years on SSI rates.

\section{Results}

A total of 4703 records were identified through the databases' search, after duplicates were removed, of which the full text of 172 was reviewed, and 16 were included in our systematic review (Fig. 1). No additional article was included following backward citation tracking. Six studies were from the United States (U. S.), two from Italy, two from Spain, one each from Australia, China, Germany, the Netherlands and Switzerland, and one was an international study conducted across Australia, Singapore, South Korea and 12 European countries. Apart from the Dutch study, published in 2008, the remaining 15 were published in the last decade, between 2011 and 2021, with data collected between 1996 and 2016. The 16 studies included comprised 1,314,608 colorectal procedures, and are described in detail in Table 1. SSI incidence ranged from 3.2 to $27.6 \%$, and the methodological quality score varied between 11 and 25 .

Figure 2 summarizes the main findings per hospital determinant. Six studies [11, 15-19] addressed structural variables-hospital size, ownership, affiliation and being an oncological hospital. Two out of the five evaluating hospital size adjusted their analysis for patient and procedural risk factors, finding that larger hospitals were either associated with higher SSI [16] or had no association [18]. In Germany, ownership type was not associated with SSI following colon procedures [18]. In the U. S., crude estimates suggest that hospitals with medical school affiliation were associated with higher incisional and organ/space SSI [11] compared with hospitals without that affiliation, whilst oncological hospitals in the same country were associated with higher superficial SSI incidence (but not organ/space) compared to non-specialized hospitals, independently of patient demographics, procedural risk factors and surgical complexity [19].

Five studies addressed how hospital or surgeon caseload associates with SSI. Hospital volume was defined as the annual volume of colorectal surgeries performed in hospitals [20], the average annual number of rectal procedures [21] or the colectomy case volume only [22]. All presented crude estimates, each reaching a different conclusion (Fig. 2). One study concluded that less experienced surgeons were associated with more postsurgical complications-SSI and others [23], while in the other no significant difference was found between high and low volume surgeons, though medium volume surgeons had significantly less SSIs than high volume ones [24]. Crude analysis suggests that higher safety-net burden may be associated with increased SSI rates [22], and the study evaluating post-discharge destinations found no difference in SSI rates between patients discharged home versus patients discharged to skilled facilities, after adjusting for 19 endogenous and exogenous risk factors [25].

The impact of surveillance over time on SSI rates was evaluated in four studies. In a large international study from 2019, each additional year of surveillance was associated with a lower SSI frequency, using the former year as reference. Additionally, participating in a surveillance network for over five years was associated with lower SSI rates [26]. The same conclusions were found in Italy, in the same year [27], although in the Netherlands, in 2008, no association was found [28]. Contrarily to these findings, one study using data from the Swiss surveillance system showed that time from the start of surveillance to the operation was significantly associated with higher SSI rates in colorectal surgery [29]. All surveillance analyses were adjusted for patient and procedure variables, and are shown in Table 1; Fig. 2.

\section{Discussion}

Although it has been recognized for over 20 years that hospital characteristics may be associated with SSI [5], as they have been shown with other adverse events [30-32], we found few studies addressing them. SSI rates also showed a wide range in incidence, though most use the $\mathrm{CDC}$ criteria, suggesting that case identification, as 


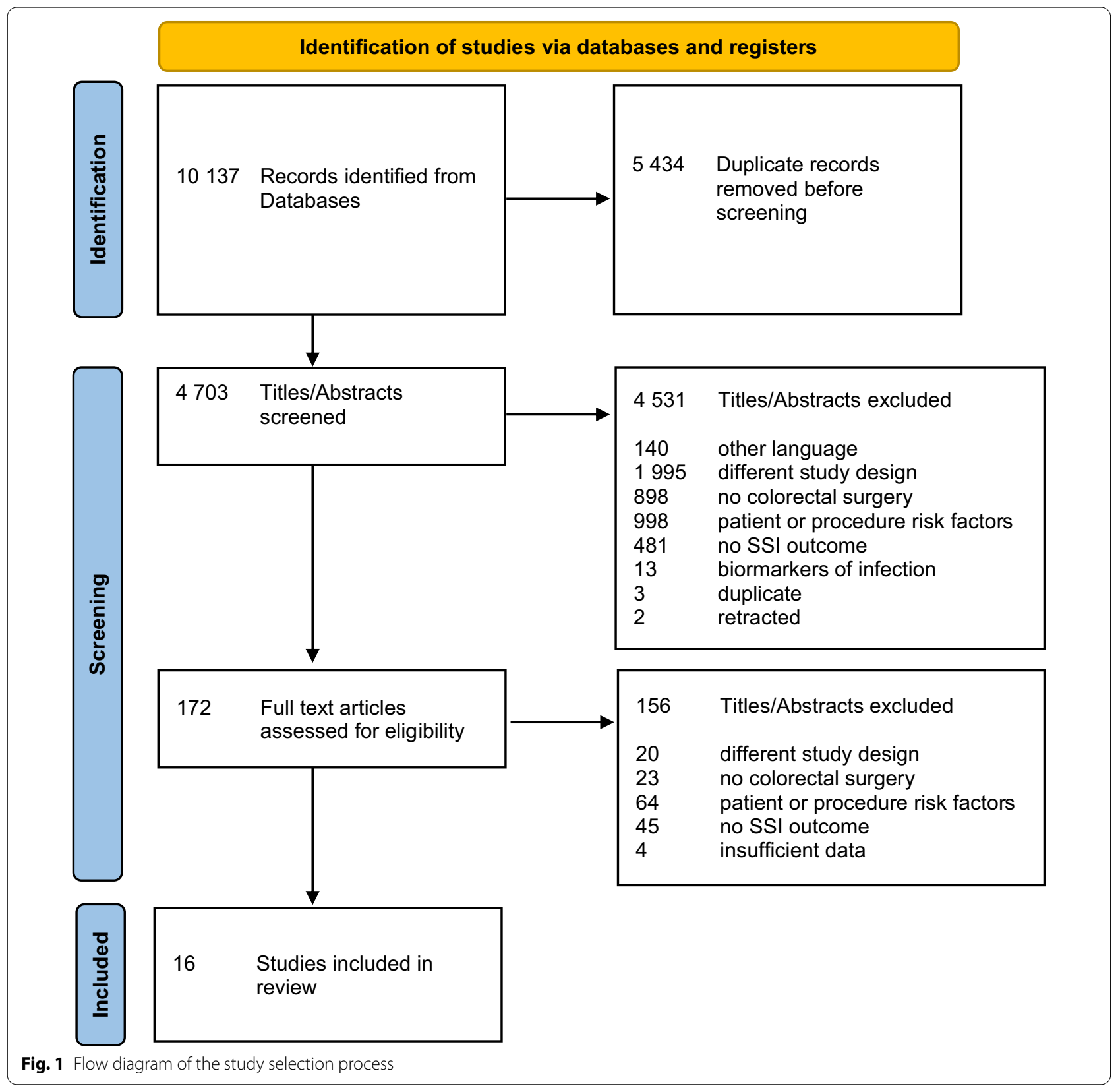

well as follow-up time, may be markedly different across settings, a major issue to be addressed given that SSI incidence is commonly used as a quality indicator for benchmarking between institutions and countries. Moreover, most data retrieved by this review is based on crude estimates, and needs to be interpreted with caution.

Public or private ownership had no apparent association with SSI after colorectal surgery in the German setting, although public hospitals had significantly less SSI after hip prosthesis following arthrosis [18]. A paper from Switzerland, albeit not providing sufficient data for the estimation of measures of association-and thus failing to meet our inclusion criteria-claimed private hospitals had fewer SSI after colorectal surgery [33]. In Australia, a study reported that private hospitals invest significantly less than public institutions in surveillance resources, emphasizing the possible underreporting of infections in the former setting [34]. While the meaning of private and public hospitals is similar throughout the world, the population served, the types of procedures performed, the structural and processual characteristics of hospitals and the financial incentives may differ 


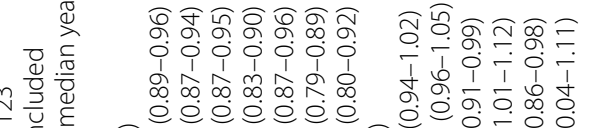

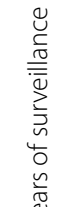

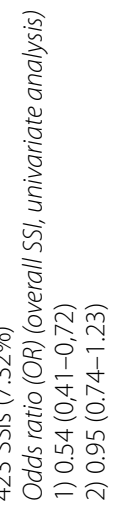

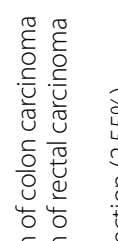

है

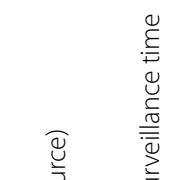

हैं

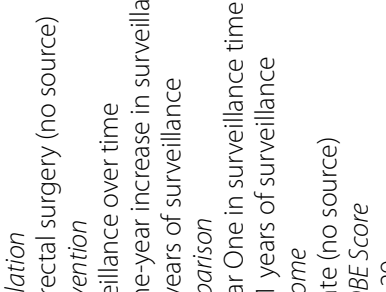

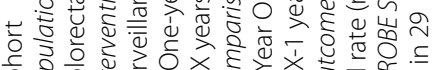

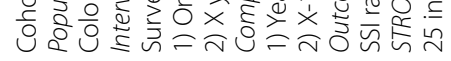
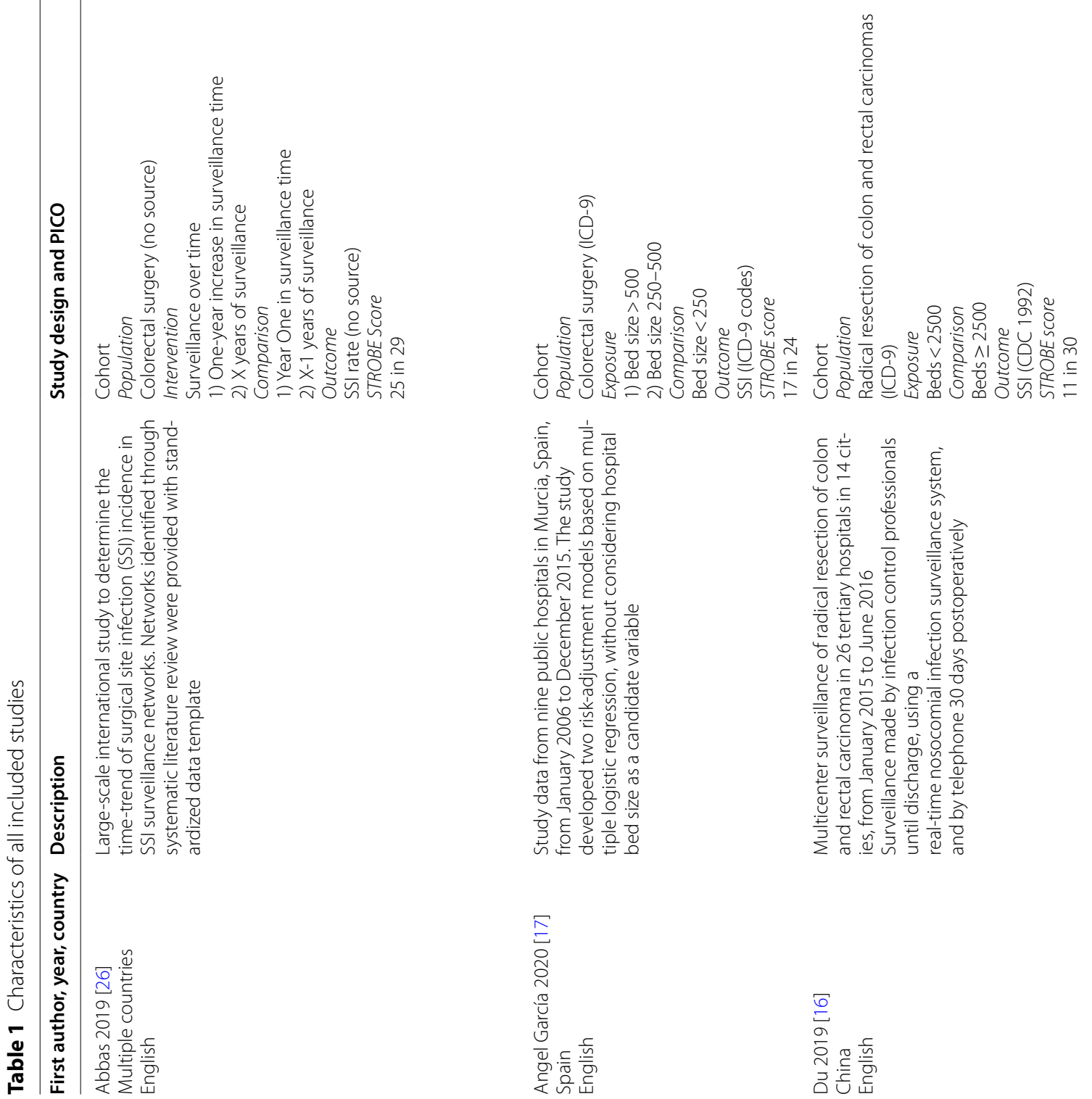


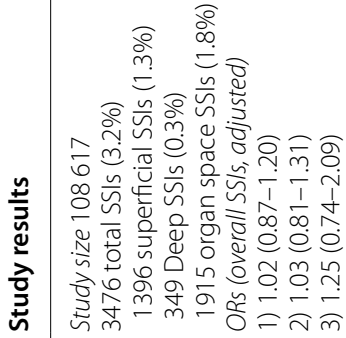

高

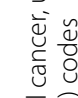

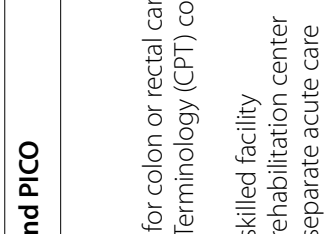

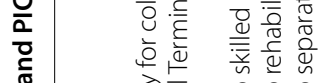

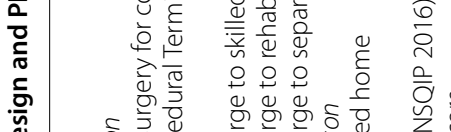

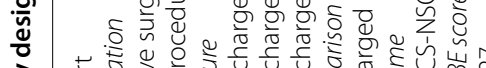

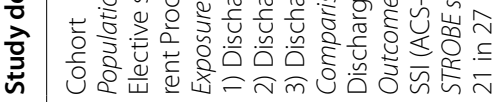

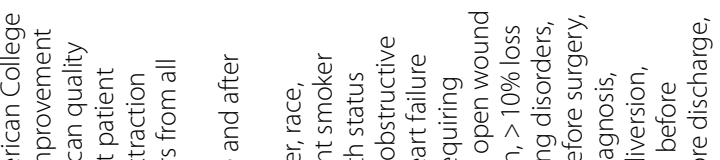

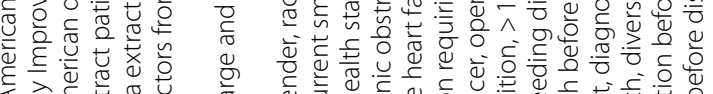

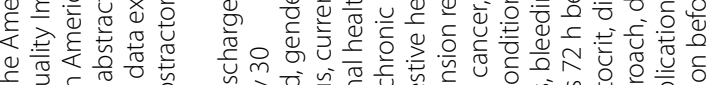

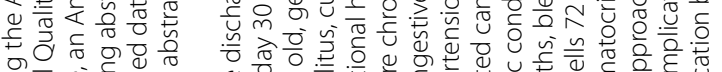

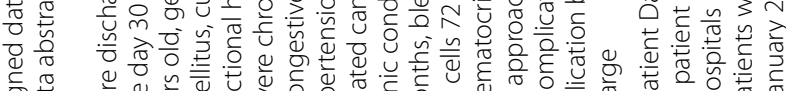

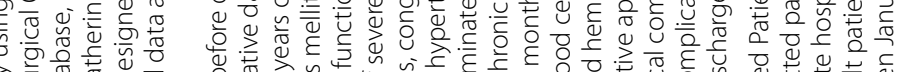

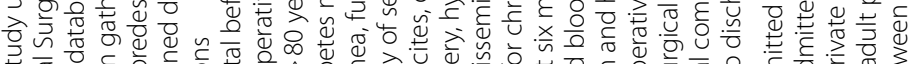

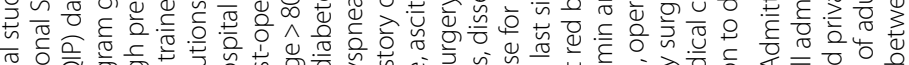

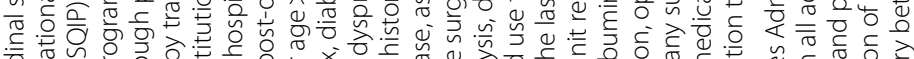

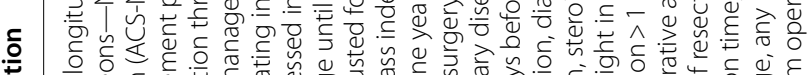

흔

贾
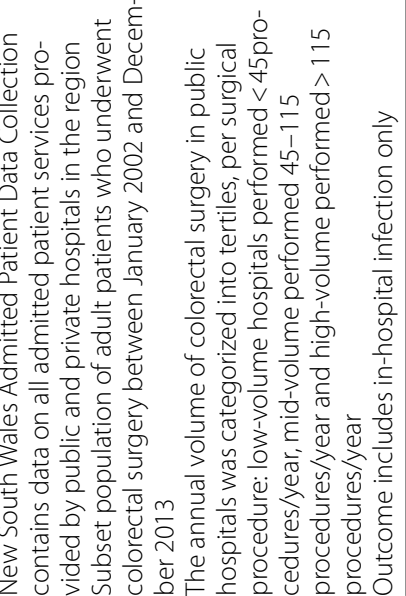

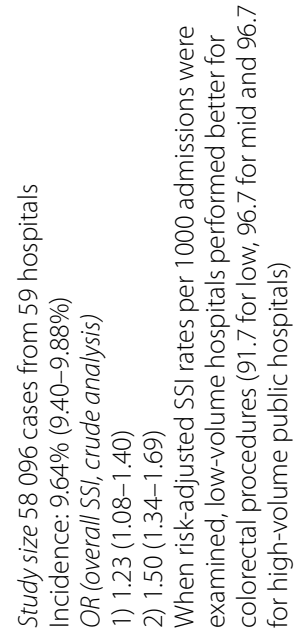

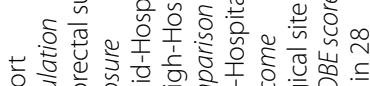

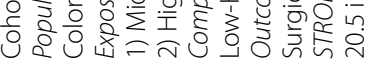




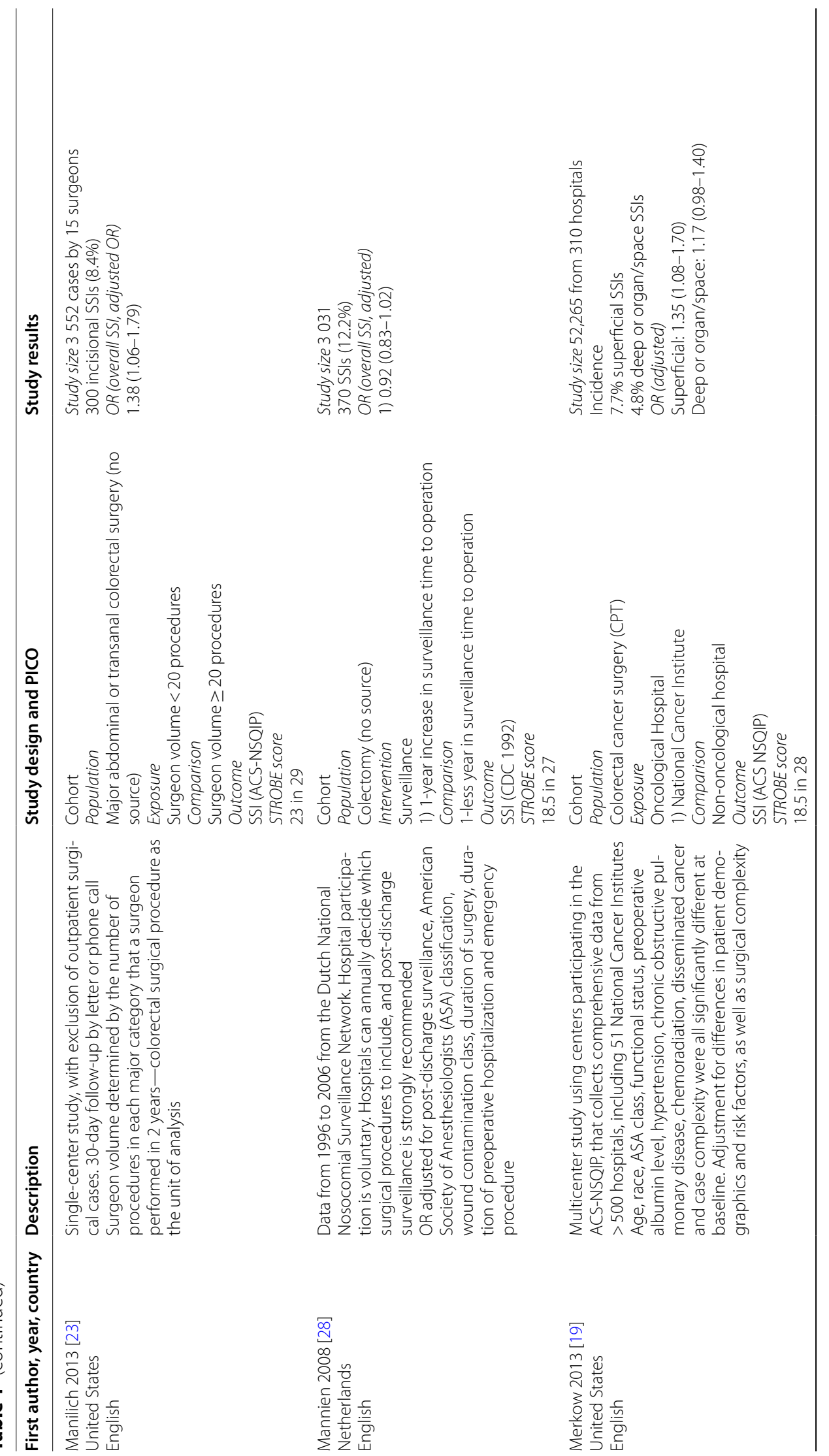




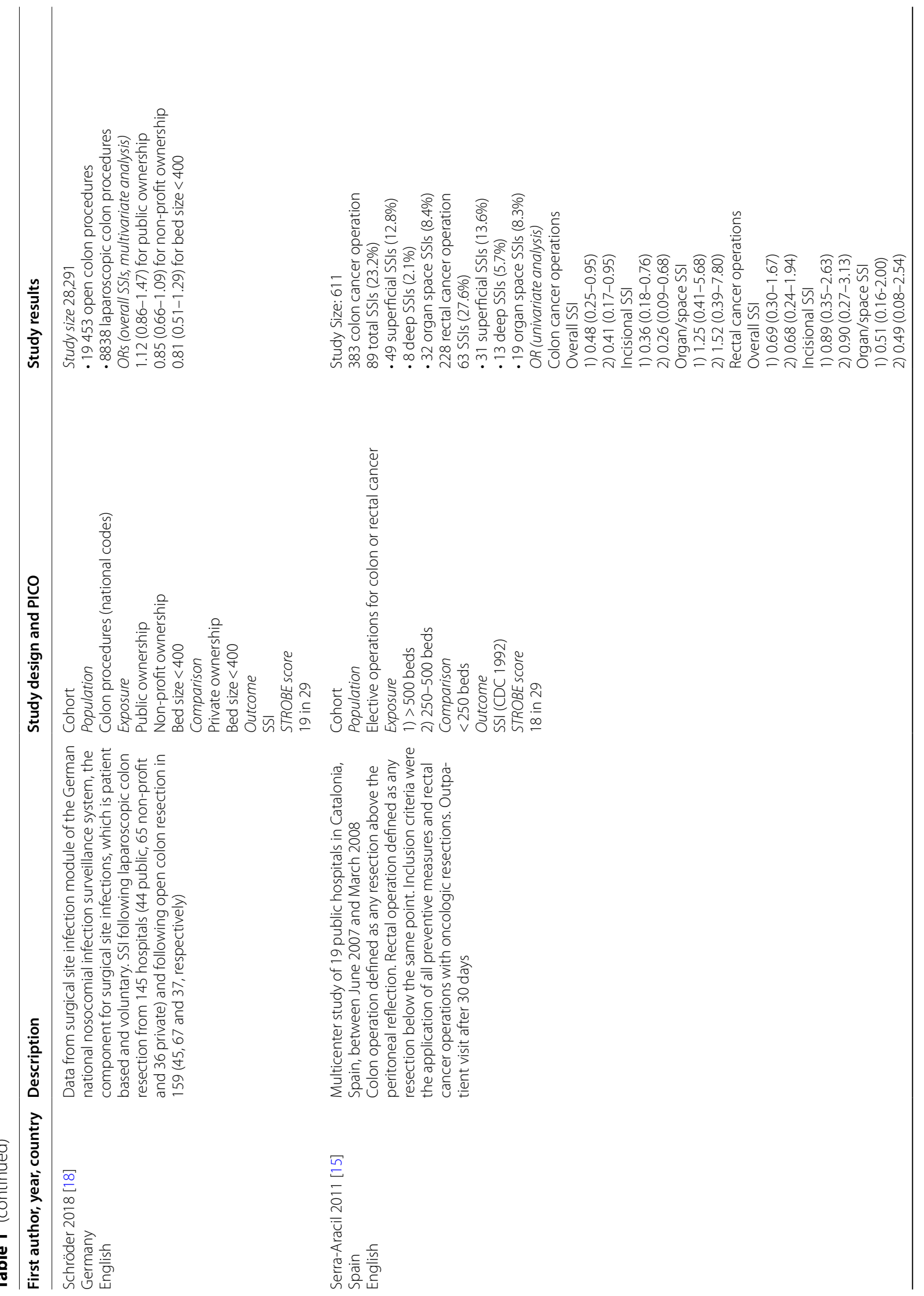




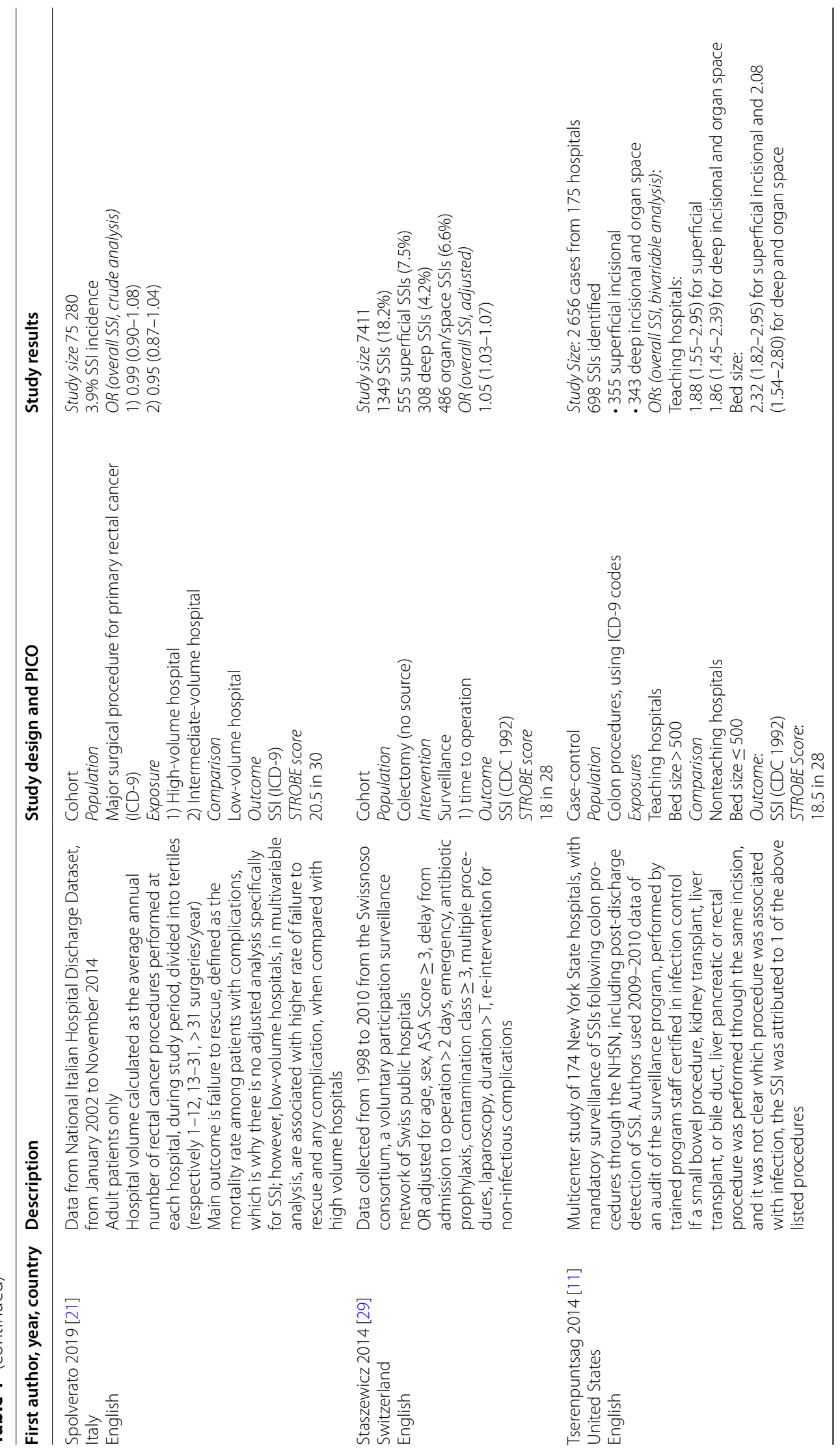




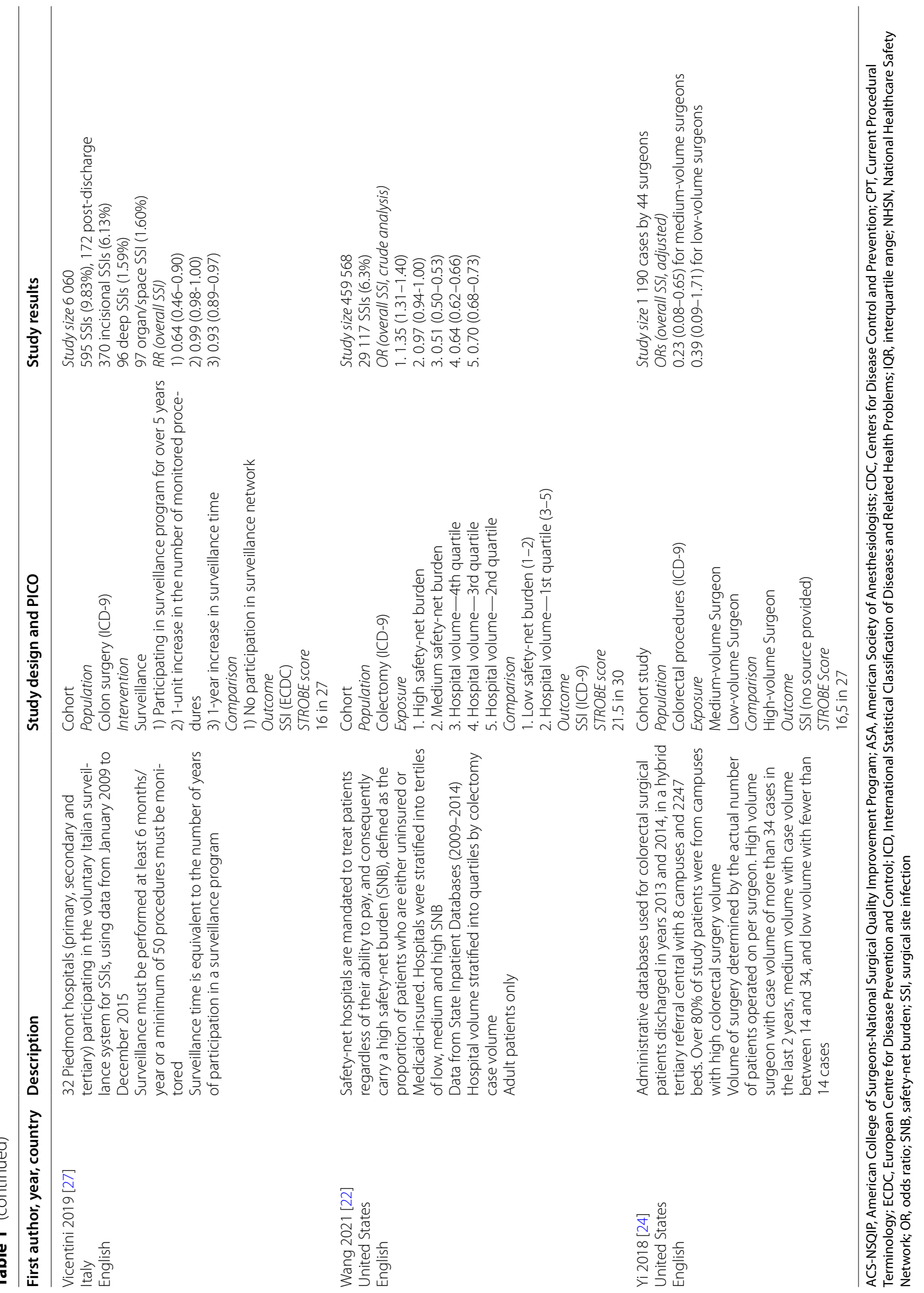


considerably between countries, precluding the external validity of these conclusions.

The most analyzed hospital characteristic was hospital size. Only two studies $[16,18]$ provided adjusted ORs for patient and procedure factors, and both considered different cutoff points than their counterparts, who used 500 beds [11, 15, 17], as proposed by the NHSN risk adjustment methodology [6]. One found no evidence of association using 400 beds as a cutoff, though it did find an association between hospital size and all device-associated and ventilator-associated infection, central venous catheter-bloodstream infection, infection by Clostridioides difficile and methicillin-resistant Staphylococcus aureus [18]. The other concluded that larger hospitals have significantly higher SSI after colorectal surgery, yet it used 1500 and 2500 beds as cutoffs, so the finding may yield no meaning in most countries of the world [16]. Comparisons among countries are also limited for oncological hospitals. While most countries dispose of specialized hospitals in cancer care, National Cancer Institutes are specific to the U. S., as they have a different payment mechanism than other American hospitals and are exempt from reporting all process-of-care and outcome measures to the Centers for Medicare \& Medicaid Services [35]. Previously defined safety-net burden is also U. S.-specific. In this case, associations may also be strongly affected by patient case mix. The authors did find a significant association between high burden and in-hospital mortality and general complications, but, unfortunately, no adjusted analysis was conducted disaggregated at the SSI level [22]. Although no difference was found for SSI, discharge to skilled facilities was associated with higher respiratory morbidity, sepsis and vascular thromboembolism [25]. It has been suggested that most SSIs occur after discharge and, thus, may be affected by post-care variables [36], yet we found no other study addressing them.

The three papers on hospital volume [20-22], defined by specific colorectal procedures, provided crude data only. Furthermore, two used ICD-10 to detect in-patient SSI [20, 21], probably underreporting SSI incidence since administrative data has been shown to have limited accuracy for the detection of SSI [37]. Regarding surgeon caseload, the study failing to find an association acknowledged that the small number case may have been insufficiently robust [24].

The positive impact of surveillance on SSI has been widely documented, although many studies focus on total or non-colorectal procedures [38-40], not accounting for the specificities of each SSI. It is accepted that surveillance may decrease SSI rates through two mechanisms: feedback and/or surveillance effect, similar to the Hawthorne effect [41]. At the same time, an artificial increase in the SSI rate may occur, due to changes in case identification, including better registry of previously unreported infections and active post-discharge case finding [36, 42], and due to changes in case mix over time [43]. This artificiality is well supported by a recent study that found a positive correlation between infection rates and audit quality [33], following the biblical sermon: "seek, and ye shall find" [44]. Two papers found that each one-year increase in surveillance time was associated with reduced SSI after colorectal surgery, while one paper failed to find any association. Both positive effects were marginal [0.93 (95\%CI 0.89-0.97) in one study [27] and 0.84 (0.79-0.89) in the best year of the other [26]], and could have limited clinical relevance. Relevantly, the influence of the surveillance effect and better case finding tends to wane over time. Hence, both papers concluding that the impact of surveillance is better noticed after the fifth year of its implementation support the impact of feedback on SSI incidence [26, 27]. Longer time trends may be needed to obtain more accurate results, even if an independent effect may exist by hospitals joining surveillance networks at a later point in time, benefiting from national efforts and overall better practices [26]. As opposed to this, one paper found that the longer the time from surveillance to procedure, the higher the SSI rate after colorectal surgery, as well as after appendectomy and knee arthroplasty [29]. Influencing these disparate findings is the fact that some surveillance networks make it mandatory for hospitals to participate, while others have voluntary participation. In the latter, there may be a selection bias similar to a healthy-worker effect, as hospitals in networks tend to allocate more resources towards surveillance when compared to non-included hospitals [26]. On the other hand, participants in voluntary systems are more interested and have more time available for

(See figure on next page.)

Fig. 2 Main findings of included studies, by hospital determinant. Each column refers to a single study. The number on top of each column is the STROBE classification of the study, and the number below is the year it was published. a maximum STROBE score of 29, $\mathbf{b}$ maximum STROBE score of 24, c maximum STROBE score of 28, $\mathbf{d}$ maximum STROBE score of 30, e maximum STROBE score of 27. Black columns refer to adjusted associations, grey refer to crude. Full columns refer to overall SSI as outcome, horizontal strips refer to superficial infection and diagonal strips to deep and organ/space infections 


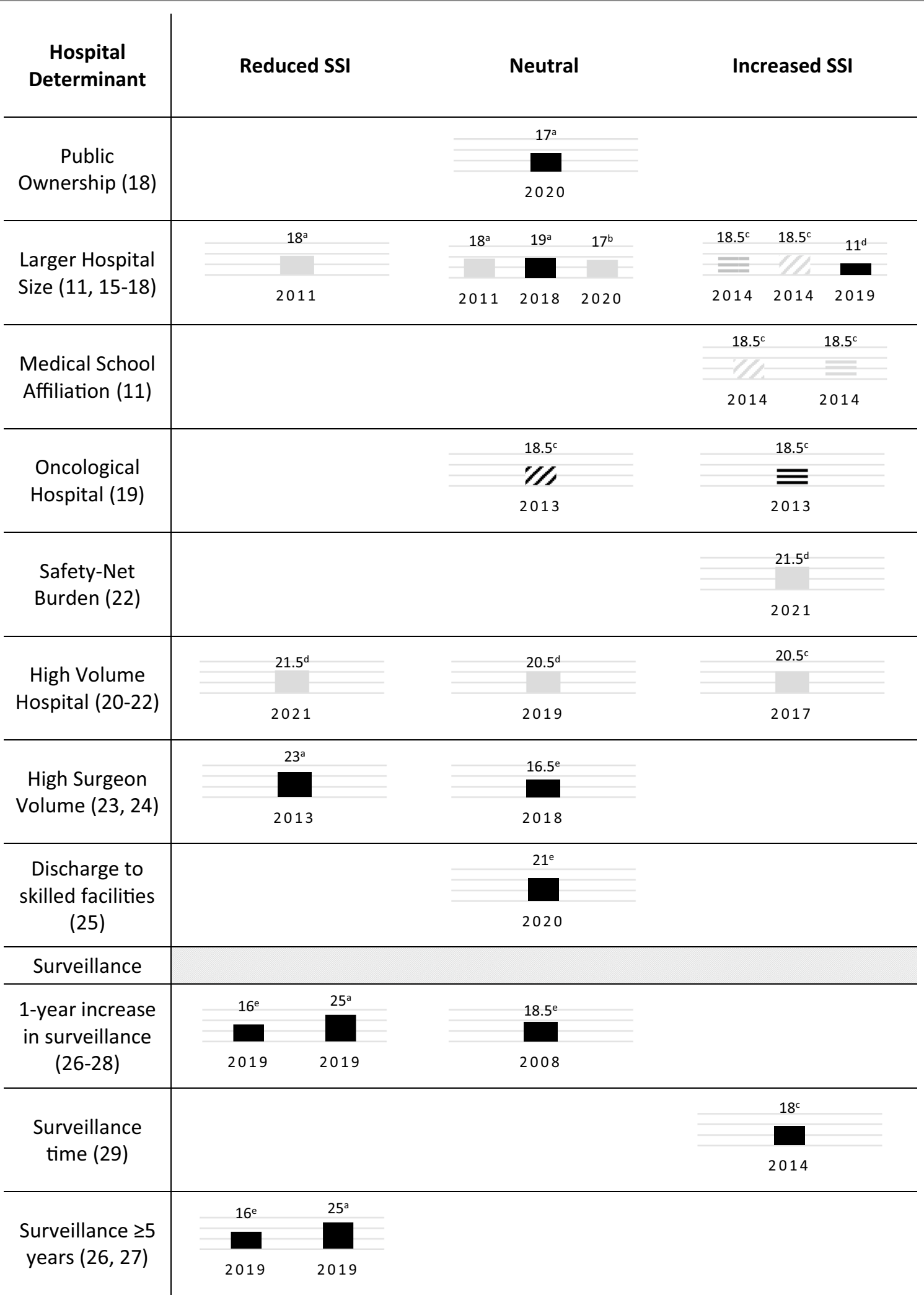

Fig. 2 (See legend on previous page.) 
surveillance, and thus are more likely to produce more accurate data [45].

To the best of our knowledge, no systematic review has been published regarding the association between hospital characteristics and SSI. Our search strategy aimed at maximum sensitivity, focusing on three major databases that retrieved a large volume of initial results. It is unlikely that relevant papers were not retrieved as all included studies were written in English and no additional manuscript was found through backward citation. By focusing on SSI after colorectal surgery, we excluded papers evaluating surveillance on SSI as whole. Using the STROBE statement, we found that most papers failed to address how missing data was handled (14 in 16), and to clarify the study's design in the title or abstract (13 in 16). While study limitations were almost ubiquitously described, they tended to lack the description of the direction and magnitude of identified biases. Due to the heterogeneity found across studies, even when analyzing the same risk factor, we were unable to quantitatively combine study findings in a meta-analysis. Many relevant healthcare delivery variables were not reviewed as we failed to find any study addressing them-that would be the case of nurse staffing, rurality or whether hospitals had an infection control team. Many hospital factors may be highly correlated, as teaching hospitals tend to be larger, urban and have a higher caseload. Healthcare delivery-and its outcomes-is also dependent on regional and national regulations, incentives and the health literacy of the population. Finally, we addressed colorectal surgery as a whole, because most colorectal surgeons perform both colon and rectal procedures. However, they appear to have different SSI rates and, quite possibly, different risk factors [39], and thus it would be relevant to consider studying them as different entities in the future.

\section{Conclusions}

Although there is a paucity of studies addressing hospital-level factors on SSI, surgeon experience and the implementation of surveillance appear to be associated with better outcomes. In order for hospitals and services to be efficiently optimized, more studies addressing these variables are needed that take into account the confounding effect of patient case mix.

\footnotetext{
Abbreviations

CDC: Centers for Disease Control and Prevention; Cl: Confidence Interval; HAI: Healthcare-Associated Infection; ICD10: International Statistical Classification of Diseases and Related Health Problems, 10th Revision; NHSN: National Healthcare Safety Network; OR: Odds Ratio; RR: Relative risk; SSI: Surgical site infection; STROBE: Strengthening the Reporting of Observational Studies in Epidemiology.; U. S.: United States..
}

\section{Acknowledgements} Not applicable.

\section{Authors' contributions}

$\mathrm{RM}$ and $\mathrm{BP}$ were responsible for data acquisition and application of inclusion criteria. RM was responsible for data analysis and drafted the work. All authors contributed to the conception and design of the work, interpretation of data and substantial revision of each draft. All authors approved the submitted version, and agree both to be accountable for the author's own contributions and to ensure that questions related to the accuracy or integrity of any part of the work are appropriately investigated, resolved and documented. All authors read and approved the final manuscript.

\section{Funding}

The Epidemiology Research Unit (EPIUnit, Unidade de Investigação em Epidemiologia_-Instituto de Saúde Pública da Universidade do Porto) is funded by the Portuguese Foundation for Science and Technology (POCI-01-0145FEDER-006862; Ref. (UIDB/04650/2020).

\section{Availability of data and materials}

All data generated or analyzed during this study are included in this published article.

\section{Declarations}

\section{Ethics approval and consent to participate}

The protocol of this study was submitted and approved by the Ethics Committee of Instituto de Saúde Pública da Universidade do Porto (CE20171), in November 2020. No informed consent was deemed necessary.

\section{Consent for publication \\ Not applicable.}

\section{Competing interests \\ The authors declare that they have no competing interests.}

\section{Author details}

${ }^{1}$ EPIUnit—Instituto de Saúde Pública, Universidade do Porto, Rua das Taipas 135, 4050-091 Porto, Portugal. ${ }^{2}$ Department of Public Health and Forensic Sciences and Medical Education, Faculdade de Medicina, Universidade do Porto (University of Porto Medical School), Porto, Portugal. ${ }^{3}$ Laboratory for Integrative and Translational Research in Population Health (ITR), Porto, Portugal.

Received: 6 August 2021 Accepted: 7 September 2021

Published online: 30 September 2021

\section{References}

1. Magill S, Hellinger W, Cohen J. Prevalence of healthcare-associated infections in acute care hospitals in Jacksonville, Florida. Infect Control Hosp Epidemiol. 2012;33:1229-30.

2. Umscheid CA, Mitchell MD, Doshi JA, Agarwal R, Williams K, Brennan PJ. Estimating the proportion of healthcare-associated infections that are reasonably preventable and the related mortality and costs. Infect Control Hosp Epidemiol. 2011;32(2):101-14.

3. Badia JM, Casey AL, Petrosillo N, Hudson PM, Mitchell SA, Crosby C. Impact of surgical site infection on healthcare costs and patient outcomes: a systematic review in six European countries. J Hosp Infect. 2017;96(1):1-15.

4. European Centre for Disease Prevention and Control. Healthcareassociated infections: surgical site infections. Annual Epidemiological Report for 2017.

5. Mangram AJ, Horan TC, Pearson ML, Silver CS, Jarvis W. Guideline for the Prevention of Surgical Site Infection. Infect Control Hosp Epidemiol. 1999;20(4):247-79.

6. The NHSN Standardized Infection Ratio (SIR). A Guide to the SIR. https://www.cdc.gov/nhsn/pdfs/ps-analysis-resources/nhsnsir-guide. pdf. Accessed June 2021 
7. Seidelman JL, Lewis SS, Baker AW, Anderson DJ. Surgical Site Infections. In: Weber DJ, Talbot TR, editors. Mayhall's Hospital Epidemiology and Infection Prevention Fifth Edition: Wolters Kluwer; 2021. p. 183-97.

8. World Health Organization. (2018). Global guidelines for the prevention of surgical site infection, 2nd ed. World Health Organization. https:// apps.who.int/iris/handle/10665/277399 (Last accessed May 1, 2021).

9. Pop-Vicas AE, Abad C, Baubie K, Osman F, Heise C, Safdar N. Colorectal bundles for surgical site infection prevention: a systematic review and meta-analysis. Infect Control Hosp Epidemiol. 2020:1-8.

10. Berrios-Torres SI, Umscheid CA, Bratzler DW, Leas B, Stone EC, Kelz RR, et al. Centers for disease control and prevention guideline for the prevention of surgical site infection, 2017. JAMA Surg. 2017;152(8):784-91.

11. Tserenpuntsag B, Haley V, Van Antwerpen C, Doughty D, Gase KA, Hazamy PA, et al. Surgical site infection risk factors identified for patients undergoing colon procedures, New York State 2009-2010. Infect Control Hosp Epidemiol. 2014;35(8):1006-12.

12. Higgins J, Green S. Cochrane handbook for systematic reviews of interventions. Chichester: Wile; 2011

13. Moher D, Liberati A, Tetzlaff J, Altman DG. Preferred reporting items for systematic reviews and meta-analyses: the PRISMA statement. PLoS Med. 2009;6(7)

14. von Elm E, Altman DG, Egger M, Pocock SJ, Gotzsche PC, Vandenbroucke $J$ P, et al. The Strengthening the Reporting of Observational Studies in Epidemiology (STROBE) statement: guidelines for reporting observational studies. J Clin Epidemiol. 2008;61 (4):344-9.

15. Serra-Aracil X, García-Domingo MI, Parés D, Espin-Basany E, Biondo S, Guirao X, et al. Surgical site infection in elective operations for colorectal cancer after the application of preventive measures. Arch Surg. 2011;146(5):606-12

16. Du M, Liu B, Li M, Cao J, Liu D, Wang Z, et al. Multicenter surveillance study of surgical site infection and its risk factors in radical resection of colon or rectal carcinoma. BMC Infect Dis. 2019;19(1):411.

17. Angel García D, Martínez Nicolás I, García Marín JA, Soria Aledo V. Riskadjustment models for clean and colorectal surgery surgical site infection for the Spanish health system. Int I Qual Health Care. 2020;32(9):599-608.

18. Schröder C, Behnke M, Geffers C, Gastmeier P. Hospital ownership: a risk factor for nosocomial infection rates? J Hosp Infect. 2018;100(1):76-82.

19. Merkow RP, Bentrem DJ, Chung JW, Paruch JL, Ko CY, Bilimoria KY. Differences in patients, surgical complexity, and outcomes after cancer surgery at National Cancer Institute-designated cancer centers compared to other hospitals. Med Care. 2013;51(7):606-13.

20. Furuya-Kanamori L, Doi SAR, Smith PN, Bagheri N, Clements ACA, Sedrakyan A. Hospital effect on infections after four major surgical procedures: outlier and volume-outcome analysis using all-inclusive state data. J Hosp Infect. 2017;97(2):115-21.

21. Spolverato G, Gennaro N, Zorzi M, Rugge M, Mescoli C, Saugo M, et al. Failure to rescue as a source of variation in hospital mortality after rectal surgery: The Italian experience. Eur J Surg Oncol. 2019;45(7):1219-24.

22. Wang W, Hoyler MM, White RS, Tangel VE, Pryor KO. Hospital safety-net burden is associated with increased inpatient mortality and perioperative complications after colectomy. J Surg Res. 2021;259:24-33.

23. Manilich E, Vogel JD, Kiran RP, Church JM, Seyidova-Khoshknabi D, Remz FH. Key factors associated with postoperative complications in patients undergoing colorectal surgery. Dis Colon Rectum. 2013;56(1):64-71.

24. Yi D, Monson JRT, Stankiewicz CC, Atallah S, Finkler NJ. Impact of colorectal surgeon case volume on outcomes and applications to quality improvement. Int J Colorectal Dis. 2018;33(5):635-44.

25. Abd El Aziz MA, Grass F, Behm KT, D'Angelo AL, Mathis KL, Dozois EJ, et al. How skilled are skilled facilities? Post-discharge complications after colorectal cancer surgery in the U.S. Am J Surg. 2020.

26. Abbas M, de Kraker MEA, Aghayev E, Astagneau P, Aupee M, Behnke $M$, et al. Impact of participation in a surgical site infection surveillance network: results from a large international cohort study. J Hosp Infect. 2019;102(3):267-76

27. Vicentini C, Dalmasso P, Politano G, Furmenti MF, Quattrocolo F, Zotti CM. Surgical site infections in Italy, 2009-2015: incidence, trends, and impact of surveillance duration on infection risk. Surg Infect (Larchmt). 2019;20(6):504-9.
28. Mannien J, van den Hof S, Muilwijk J, van den Broek PJ, van Benthem B, Wille JC. Trends in the incidence of surgical site infection in the Netherlands. Infect Control Hosp Epidemiol. 2008;29(12):1132-8.

29. Staszewicz W, Eisenring MC, Bettschart V, Harbarth S, Troillet N. Thirteen years of surgical site infection surveillance in Swiss hospitals. J Hosp Infect. 2014;88(1):40-7.

30. Cots F, Mercade L, Castells X, Salvador X. Relationship between hospital structural level and length of stay outliers. Implications for hospital payment systems. Health Policy. 2004;68(2):159-68.

31. Pieper D, Mathes T, Neugebauer E, Eikermann M. State of evidence on the relationship between high-volume hospitals and outcomes in surgery: a systematic review of systematic reviews. J Am Coll Surg. 2013;216(5):1015-25 e18.

32. Horwitz LI, Wang Y, Altaf FK, Wang C, Lin Z, Liu S, et al. Hospital characteristics associated with postdischarge hospital readmission, observation, and emergency department utilization. Med Care. 2018;56(4):281-9.

33. Atkinson A, Eisenring MC, Troillet N, Kuster SP, Widmer A, Zwahlen M, et al. Surveillance quality correlates with surgical site infection rates in knee and hip arthroplasty and colorectal surgeries: a call to action to adjust reporting of SSI rates. Infect Control Hosp Epidemiol. 2021. 1-7.

34. Mitchell BG, Hall L, MacBeth D, Gardner A, Halton K. Hospital infection control units: staffing, costs, and priorities. Am J Infect Control. 2015;43(6):612-6.

35. Merkow RP, Yang AD, Pavey E, Song MW, Chung JW, Bentrem DJ, et al. Comparison of hospitals affiliated with PPS-exempt cancer centers, other hospitals affiliated with NCI-designated cancer centers, and other hospitals that provide cancer care. JAMA Intern Med. 2019;179(8):1043-51.

36. Woelber E, Schrick EJ, Gessner BD, Evans HL. Proportion of surgical site infections occurring after hospital discharge: a systematic review. Surg Infect (Larchmt). 2016;17(5):510-9.

37. van Mourik MS, van Duijn PJ, Moons KG, Bonten MJ, Lee GM. Accuracy of administrative data for surveillance of healthcare-associated infections: a systematic review. BMJ Open. 2015;5(8):e008424.

38. Geubbels E, Nagelkerke J, Groot J, Vandenbroucke-Grauls CM, Grobbee D, De Boer AS. Reduced risk of surgical site infections through surveillance in a network. Int J Quality Health Care. 2006;18:2127-133.

39. Konishi T, Watanabe T, Morikane K, Fukatsu K, Kitayama J, Umetani N, et al. Prospective surveillance effectively reduced rates of surgical site infection associated with elective colorectal surgery at a university hospital in Japan. Infect Control Hosp Epidemiol. 2006;27(5):526-8.

40. Chungsiriwattana W, Sangkhathat S, Kongkamol C, Suwalak N, Phainuphong $\mathrm{P}$, Komet $\mathrm{O}$, et al. Decreasing trend of surgical site Infections among surgical patients in a University Hospital in Thailand after an active surveillance program. Surg Infect (Larchmt). 2019;20(5):382-9.

41. Gastmeier P, Schwab F, Sohr D, Behnke M, Geffers C. Reproducibility of the surveillance effect to decrease nosocomial infection rates. Infect Control Hosp Epidemiol. 2009;30(10):993-9.

42. Troillet N, Aghayev E, Eisenring MC, Widmer AF. First results of the Swiss national surgical site infection surveillance program: who seeks shall find. Infect Control Hosp Epidemiol. 2017;38(6):697-704.

43. Abbas M, de Kraker MEA, Aghayev E, Astagneau P, Aupee M, Behnke $M$, et al. Impact of participation in a surgical site infection surveillance network: results from a large international cohort study. J Hosp Infect. 2019;102(3):267-76.

44. King James Version Bible, 2009, Matt, 7:7.

45. Leaper D, Tanner J, Kiernan M. Surveillance of surgical site infection: more accurate definitions and intensive recording needed. J Hosp Infect. 2013;83(2):83-6.

\section{Publisher's Note}

Springer Nature remains neutral with regard to jurisdictional claims in published maps and institutional affiliations 